\title{
POLARIZATION ANGLE SWINGS REDISCUSSED
}

\author{
W. Sieber \\ Max-Planck-Institut für Radioastronomie \\ Auf dem Hügel 69 \\ D-5300 Bonn 1 \\ Federal Republic of Germany
}

The polarization angle swing observed in some pulsars, e.g. PSR 0833-45, may be understood as the pattern which results when our line of sight cuts through the magnetic dipole field of a pulsar. In such a geometry the polarization angle $\chi$ is a function of $\mu$, the angle between rotation axis and magnetic field axis and $\Delta$, the angle between magnetic field and line of sight to the observer.

For real data, $90^{\circ}$ polarization angle flips very often completely obscure smooth variations as expected from geometry. The determination of the contributions from competitive $90^{\circ}$ modes is only possible by use of data with high signal-to-noise ratio; few such mesurements exist. Our analysis is based (see also Narayan and Vivekanand, 1983) on data sets published by Backer and Rankin (1980) and Bartel et al. (1982), which we used to fit model curves as given above ( $\mu$ was kept constant: $\mu=45^{\circ}$ ).

Remarkably good results were obtained for nearly all pulsars, especially convincing for pulsars with double peaked profiles. The polarization angle swing for PSR $0823+26$ indicates that this pulsar may also be considered as a double peaked pulsar, the second component being very weak. A similar geometry might tentatively apply for PSR $1541+09$, the main component being here on the trailing side of the profile. PSR $1237+25$ and $0329+54$ show nearly central cuts through the beam $(\Delta=$ 0.2 and 0.71 respectively). It becomes clear from PSR $0329+54$ that mode-changes do obviously not influence the magnetic field geometry. Pulsars with high $\Delta$-values show very often considerable azimuthal structure in their profiles, perhaps due to absorption.

From the steepest gradient of $\chi$ it is possible to estimate an upper limit of $\Delta$, independent of $\mu$. The distribution of values gives direct evidence of detecting pulsars with a given separation between line of sight and magnetic field axis. Separations greater than $20^{\circ}$ are extremely rare. We estimate that less than $10 \%$ of all pulsars can be detected due to beaming (one beam emission). It seems that the latitudinal-longitudinal beam asymmetry is about a factor of 2 , not higher.

\section{REFERENCES}

Backer, D.C., Rankin, J.M.: 1980, Astrophys. J. Suppl. 42, 143

Bartel, N., Morris, D., Sieber, W. et al:. 1982, Astrophys. J. 258, 776

Narayan, R., Vivekanand, M.: 1983. Astron. Astrophys. 122, 45 